\title{
Conduction Disorder, CTCAE
}

National Cancer Institute

\section{Source}

National Cancer Institute. Conduction Disorder, CTCAE. NCI Thesaurus. Code C143380.

A disorder characterized by pathological irregularities in the cardiac conduction system. 